CRYSTALLOGRAPHIC COMMUNICATIONS

ISSN 2056-9890

Received 5 November 2017

Accepted 9 November 2017

Edited by J. Simpson, University of Otago, New Zealand

Keywords: crystal structure; $\mathrm{Hg}^{\text {"l }}$ compound; dipyridyl ligand; zigzag coordination polymer; hydrogen bonding; $\mathrm{C}-\mathrm{H} \cdots \pi$ interactions.

CCDC reference: 1584773

Supporting information: this article has supporting information at journals.iucr.org/e

\section{A one-dimensional $\mathrm{Hg}^{\mathrm{I}}$ coordination polymer based on bis(pyridin-3-ylmethyl)sulfane}

\author{
Suk-Hee Moon, ${ }^{a}$ Youngjin Kang ${ }^{\mathrm{b} *}$ and Ki-Min Park ${ }^{\mathrm{c} *}$
}

${ }^{a}$ Department of Food and Nutrition, Kyungnam College of Information and Technology, Busan 47011, Republic of Korea, ${ }^{\mathbf{b}}$ Division of Science Education, Kangwon National University, Chuncheon 24341, Republic of Korea, and ${ }^{\mathbf{c}}$ Research institute of Natural Science, Gyeongsang National University, Jinju 52828, Republic of Korea. *Correspondence e-mail: kangy@kangwon.ac.kr, kmpark@gnu.ac.kr

The reaction of mercury(II) chloride with bis(pyridin-3-ylmethyl)sulfane ( $L$, $\mathrm{C}_{12} \mathrm{H}_{12} \mathrm{~N}_{2} \mathrm{~S}$ ) in methanol afforded the title crystalline coordination polymer catena-poly[[dichloridomercury(II)]- $\mu$-bis(pyridin-3-ylmethyl)sulfane- $\left.\kappa^{2} N: N^{\prime}\right]$, $\left[\mathrm{HgCl}_{2} L\right]_{n}$. The asymmetric unit consists of one $\mathrm{Hg}^{\mathrm{II}}$ cation, one $L$ ligand and two chloride anions. Each $\mathrm{Hg}^{\mathrm{II}}$ ion is coordinated by two pyridine $\mathrm{N}$ atoms from separate $L$ ligands and two chloride anions. The metal adopts a highly distorted tetrahedral geometry, with bond angles about the central atom in the range 97.69 (12)-153.86 (7) ${ }^{\circ}$. Each $L$ ligand bridges two $\mathrm{Hg}^{\mathrm{II}}$ ions, forming an infinite $-(\mathrm{Hg}-L)_{n}-$ zigzag chain along the $b$ axis, with an $\mathrm{Hg} \cdots \mathrm{Hg}$ separation of 10.3997 (8) A. In the crystal, adjacent chains are connected by intermolecular $\mathrm{C}-\mathrm{H} \cdots \mathrm{Cl}$ hydrogen bonds, together with $\mathrm{Hg}-\mathrm{Cl} \cdots \pi$ interactions [chloride-tocentroid distance $=3.902$ (3) $\AA$ ], that form between a chloride anion and the one of the pyridine rings of $L$, generating a two-dimensional layer extending parallel to (101). These layers are further linked by intermolecular $\mathrm{C}-\mathrm{H} \cdots \pi$ hydrogen bonds, forming a three-dimensional supramolecular network.

\section{Chemical context}

The structural topology of coordination polymers generated from the self-assembly of transition metal ions and organic molecules functioning as spacer ligands depends mainly on the structures of the spacer ligands and the coordination geometries adopted by the metal ions. The flexibility, length and coordinating ability of the spacer ligands exert strong influences on the formation of coordination polymers and their resulting diverse topologies (Zheng et al., 2009; Leong \& Vittal, 2011; Liu et al. 2011). For this reason, both rigid and flexible dipyridyl-type spacer ligands with strong coordinating ability and functional characteristics have been widely used to construct a variety of coordination polymers with interesting structures and attractive potential applications in material science (Silva et al., 2015; Furukawa et al., 2014; Wang et al., 2012).

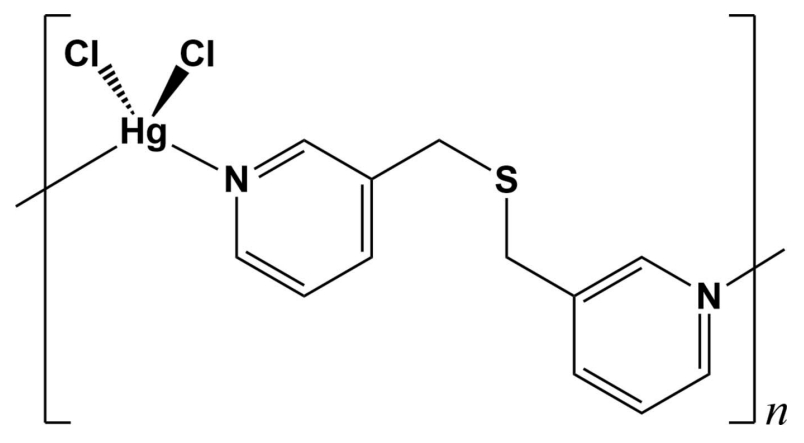


Table 1

Selected geometric parameters $\left(\AA{ }^{\circ}\right)$.

\begin{tabular}{lclr}
\hline $\mathrm{Hg} 1-\mathrm{Cl} 1$ & $2.3610(16)$ & $\mathrm{Hg} 1-\mathrm{N} 2^{\mathrm{i}}$ & $2.434(5)$ \\
$\mathrm{Hg} 1-\mathrm{Cl} 2$ & $2.3751(16)$ & $\mathrm{Hg} 1-\mathrm{N} 1$ & $2.436(5)$ \\
& & & \\
$\mathrm{Cl} 1-\mathrm{Hg} 1-\mathrm{Cl} 2$ & $153.86(7)$ & $\mathrm{Cl} 1-\mathrm{Hg} 1-\mathrm{N} 1$ & $97.69(12)$ \\
$\mathrm{Cl} 1-\mathrm{Hg} 1-\mathrm{N} 2^{\mathrm{i}}$ & $100.29(12)$ & $\mathrm{Cl} 2-\mathrm{Hg} 1-\mathrm{N} 1$ & $97.91(13)$ \\
$\mathrm{Cl} 2-\mathrm{Hg} 1-\mathrm{N} 2^{\mathrm{i}}$ & $98.03(12)$ & $\mathrm{N} 2^{\mathrm{i}}-\mathrm{Hg} 1-\mathrm{N} 1$ & $98.39(16)$ \\
\hline
\end{tabular}

Symmetry code: (i) $-x+\frac{1}{2}, y-\frac{1}{2},-z+\frac{1}{2}$.

Our group has also synthesized the flexible dipyridyl-type ligand bis(pyridine-3-ylmethyl)sulfane $(L)$, and has reported its $\mathrm{Ag}^{\mathrm{I}}$ and $\mathrm{Co}^{\mathrm{II}}$ coordination polymers (Moon et al., 2017a,b). Our continuing interest in the development of coordination polymers based on this ligand led us to investigate a coordination polymer with an $\mathrm{Hg}^{\mathrm{II}}$ cation. The reaction of mercury(II) chloride with $L$ (synthesized according to a previously reported procedure: Park et al., 2010; Lee et al., 2012) afforded the title compound. Herein, we describe its structure, which involves a one-dimensional zigzag-chain.

\section{Structural commentary}

Fig. 1 shows the molecular structure of the title compound, $\left[\mathrm{HgLCl}_{2}\right]_{n}, L=$ bis(pyridine-3-ylmethyl)sulfane, $\mathrm{C}_{12} \mathrm{H}_{12} \mathrm{~N}_{2} \mathrm{~S}$. The asymmetric unit comprises one $\mathrm{Hg}^{\mathrm{II}}$ cation, one $L$ ligand and two chloride anions. The $\mathrm{Hg}^{\mathrm{II}}$ ion is four-coordinated, binding to two $\mathrm{Cl}$ anions and two pyridine $\mathrm{N}$ atoms from two separate symmetry-related $L$ ligands, forming a highly distorted tetrahedral geometry (Fig. 1), with the tetrahedral angles falling in the range of 97.69 (12)-153.86 (7) ${ }^{\circ}$ (Table 1). The $\mathrm{S}$ atoms of the $L$ ligands are surprisingly not bound to the soft $\mathrm{Hg}^{\mathrm{II}}$ cations. Each $L$ ligand bridges two $\mathrm{Hg}^{\mathrm{II}}$ cations, resulting in an infinite zigzag chain propagating along the $b$ axis direction (Fig. 2). The separation between the $\mathrm{Hg}^{\mathrm{II}}$ ions in the chain is 10.3997 (8) $\AA$. In the $L$ ligand, the dihedral angle between the two terminal pyridine rings is $78.52(18)^{\circ}$, and the flexible thioether moiety [C4-C6-S1-C7-C8] shows a bent

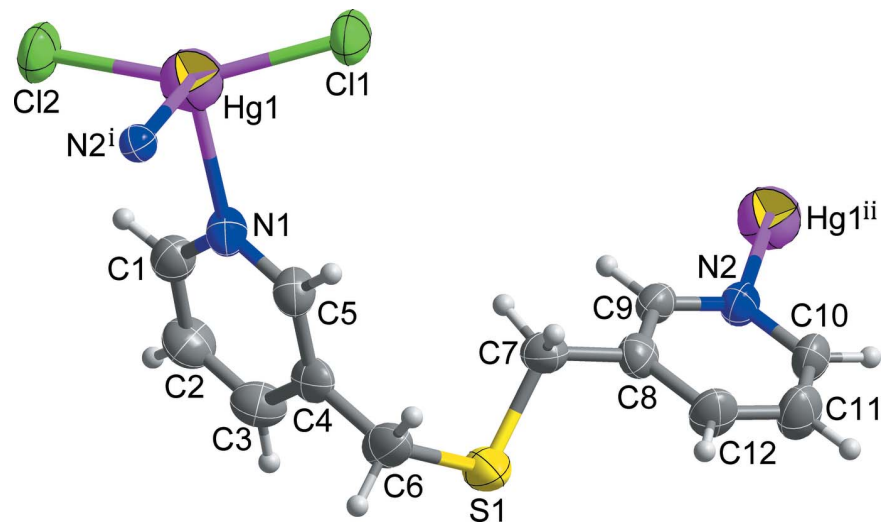

Figure 1

View of the molecular structure of the title compound, showing the atomnumbering scheme [symmetry codes: (i) $-x+\frac{1}{2}, y-\frac{1}{2},-z+\frac{1}{2}$; (ii) $-x+\frac{1}{2}$, $y+\frac{1}{2},-z+\frac{1}{2}$ ]. Displacement ellipsoids are drawn at the $50 \%$ probability level.
Table 2

Hydrogen-bond geometry $\left(\AA,^{\circ}\right)$.

$C g 2$ is the centroid of the $\mathrm{N} 2 / \mathrm{C} 8-\mathrm{C} 12$ ring.

\begin{tabular}{lllll}
\hline$D-\mathrm{H} \cdots A$ & $D-\mathrm{H}$ & $\mathrm{H} \cdots A$ & $D \cdots A$ & $D-\mathrm{H} \cdots A$ \\
\hline $\mathrm{C} 10-\mathrm{H} 10 \cdots \mathrm{C} 11^{\mathrm{ii}}$ & 0.93 & 2.80 & $3.526(6)$ & 136 \\
$\mathrm{C} 2-\mathrm{H} 2 \cdots C g 2^{\mathrm{iii}}$ & 0.93 & 2.89 & $3.689(7)$ & 145 \\
\hline
\end{tabular}

Symmetry codes: (ii) $-x,-y+1,-z$; (iii) $-x,-y+1,-z+1$.

arrangement with a gauche--anti configuration $[\mathrm{C} 4-\mathrm{C} 6-\mathrm{S} 1-$ $\left.\mathrm{C} 7=71.9(5)^{\circ} ; \mathrm{C} 6-\mathrm{S} 1-\mathrm{C} 7-\mathrm{C} 8=172.1(5)^{\circ}\right]$. The conformation of the $L$ ligand, along with its $\mathrm{N}_{\mathrm{py}}-\mathrm{Hg}-\mathrm{N}_{\mathrm{py}}$ coordination angle $\left[98.39(16)^{\circ}\right]$, may induce the zigzag topology of the chain.

\section{Supramolecular features}

In the crystal structure, adjacent zigzag chains are connected by $\mathrm{C} 10-\mathrm{H} 10 \cdots \mathrm{Cl} 1$ hydrogen bonds (Fig. 3, Table 2) and $\mathrm{Hg}-\mathrm{Cl} \cdots \pi$ interactions (Chifotides \& Dunbar, 2013; Matter et al., 2009) between the chloride anions and the pyridine rings of $L$ with $\mathrm{Cl} 2 \cdots C g 1^{\text {iv }}=3.902(3) \AA$ and $\mathrm{Hg} 1-\mathrm{Cl} 2 \cdots C g 1^{\text {iv }}=$ 77.21 (6) ${ }^{\circ}$ [Fig. 3; $C g 1$ is the centroid of the N1/C1-C5 ring; symmetry code: (iv) $-x+1,-y+1,-z+1$ ], generating layers extending parallel to (101). Neighboring layers are linked by $\mathrm{C} 2-\mathrm{H} 2 \cdots \mathrm{Cg} 2$ hydrogen bonds (Table 2; Fig. 4), resulting in the formation of a three-dimensional supramolecular network.

\section{Database survey}

A search of the Cambridge Structural Database (Version 5.38, update May 2017; Groom et al., 2016) for the title ligand (L) gave three hits. Two (REJCAL, RENHOI; Hanton et al., 2006) are copper(I) iodide coordination polymers adopting staircase- and loop-type structures, respectively. The other (EXEZOW; Seo et al., 2003) is a cyclic dimer-type silver(I) $\mathrm{BF}_{4}$ complex. Recently, our group has also reported the crystal structures of silver(I) (Moon et al., 2017a) and cobalt(II) (Moon et al., 2017b) $\mathrm{NO}_{3}$ coordination polymers that display twisted ribbon- and loop-type topologies, respectively. In these complexes, the flexible thioether moiety $\left(\mathrm{C}_{\mathrm{py}}-\mathrm{C}-\mathrm{S}-\mathrm{C}-\mathrm{C}_{\mathrm{py}}\right)$ of

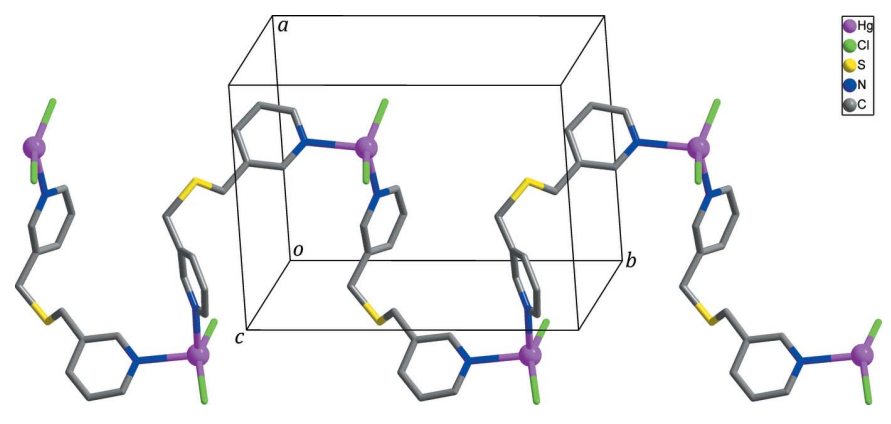

Figure 2

The polymeric zigzag chain propagating along the $b$-axis direction. $\mathrm{H}$ atoms are omitted for clarity. 


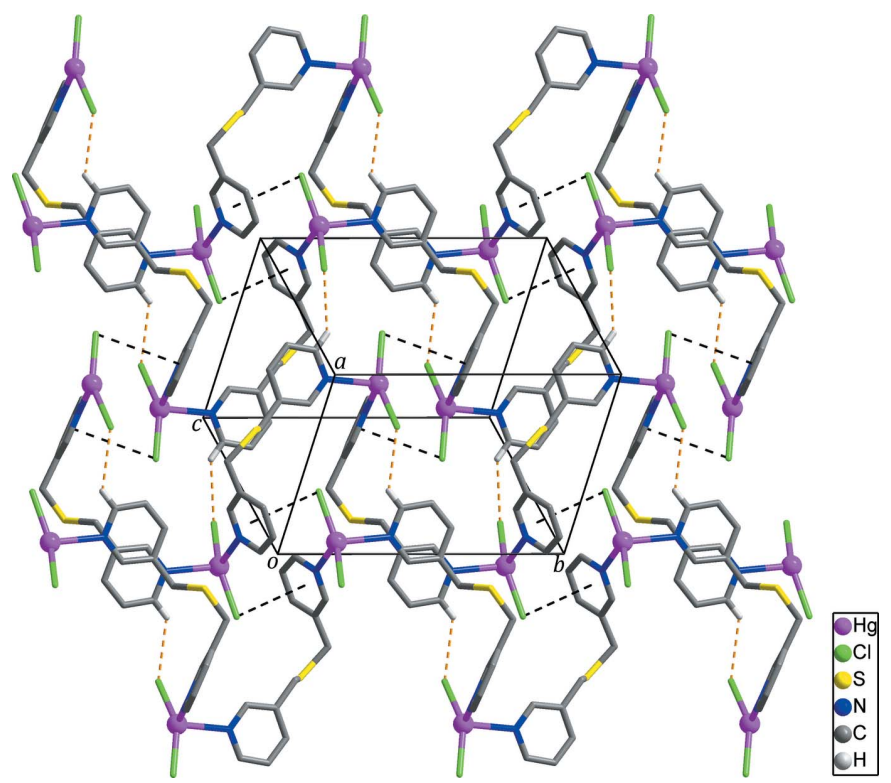

Figure 3

The layer formed through intermolecular $\mathrm{C}-\mathrm{H} \cdots \mathrm{Cl}$ hydrogen bonds (yellow dashed lines) and $\mathrm{Hg}-\mathrm{Cl} \cdots \pi$ interactions (black dashed lines) between the zigzag chains. $\mathrm{H}$ atoms not involved in intermolecular interactions are omitted for clarity.

the $L$ ligand adopts a bent arrangement that is similar to that of the $\mathrm{Hg}^{\mathrm{II}}$ polymer described here. However, the title compound displays a zigzag topology and is the first example of an $\mathrm{Hg}^{\mathrm{II}}$ coordination polymer with the ligand $L$.

\section{Synthesis and crystallization}

The $L$ ligand was synthesized according to a literature method (Park et al., 2010; Lee et al., 2012). Crystals of the title compound were obtained by slow evaporation of a methanol solution of $L$ with $\mathrm{HgCl}_{2}$ in a 1:1 molar ratio.

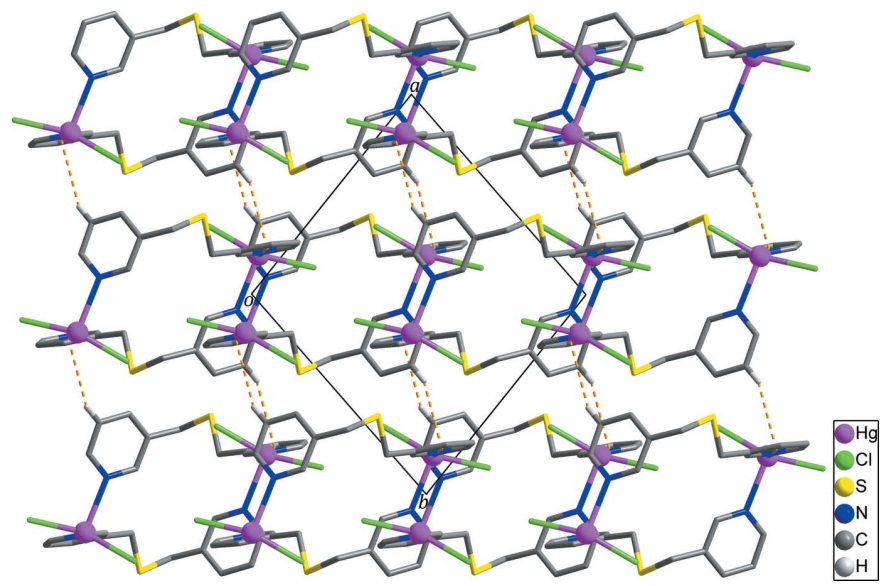

Figure 4

The three-dimensional supramolecular network generated by intermolecular $\mathrm{C}-\mathrm{H} \cdots \pi$ interactions (yellow dashed lines) between the layers of polymer chains. $\mathrm{H}$ atoms not involved in intermolecular interactions are omitted for clarity.
Table 3

Experimental details.

Crystal data

Chemical formula

$M_{\mathrm{r}}$

Crystal system, space group

Temperature (K)

$a, b, c(\AA)$

$\beta\left(^{\circ}\right)$

$V\left(\AA^{3}\right)$

$Z$

Radiation type

$\mu\left(\mathrm{mm}^{-1}\right)$

Crystal size (mm)

\author{
$\left[\mathrm{HgCl}_{2}\left(\mathrm{C}_{12} \mathrm{H}_{12} \mathrm{~N}_{2} \mathrm{~S}\right)\right]$ \\ 487.79 \\ Monoclinic, $P 2_{1} / n$ \\ 298 \\ 10.4724 (11), 13.1128 (14), \\ $10.8914(12)$ \\ $100.1171(18)$ \\ $1472.4(3)$ \\ 4 \\ Mo $K \alpha$ \\ 10.94 \\ $0.45 \times 0.40 \times 0.30$
}

Data collection

Diffractometer

Absorption correction

$T_{\min }, T_{\max }$

No. of measured, independent and observed $[I>2 \sigma(I)]$ reflections

$R_{\text {int }}$

$(\sin \theta / \lambda)_{\max }\left(\AA^{-1}\right)$

Bruker SMART APEX CCD
Multi-scan (SADABS; Bruker,
2014)
$0.447,0.746$
$8706,3197,2413$

0.047
0.639

$0.033,0.076,1.03$
3197
163
H-atom parameters constrained
$0.62,-1.62$

Refinement

$R\left[F^{2}>2 \sigma\left(F^{2}\right)\right], w R\left(F^{2}\right), S$

No. of reflections

No. of parameters

$\mathrm{H}$-atom treatment

$\Delta \rho_{\max }, \Delta \rho_{\min }\left(\mathrm{e} \AA^{-3}\right)$

$0.62,-1.62$

Computer programs: APEX2 and SAINT (Bruker, 2014), SHELXS97 and SHELXTL (Sheldrick, 2008), SHELXL2014 (Sheldrick, 2015), DIAMOND (Brandenburg, 2010) and publCIF (Westrip, 2010).

\section{Refinement}

Crystal data, data collection and structure refinement details are summarized in Table 3. All $\mathrm{H}$ atoms were positioned geometrically and refined as riding: $\mathrm{C}-\mathrm{H}=0.93 \AA$ for $\mathrm{Csp} p^{2}-$ $\mathrm{H}$ and $0.97 \AA$ for methylene $\mathrm{C}-\mathrm{H}$ with $U_{\text {iso }}(\mathrm{H})=1.2 U_{\text {eq }}(\mathrm{C})$.

\section{Funding information}

This research was supported by Basic Science Research Program through the National Research Foundation of Korea (NRF) funded by the Ministry of Education (NRF2015R1D1A3A01020410) and a 2017 Research Grant from Kangwon National University (No. 520170312).

\section{References}

Brandenburg, K. (2010). DIAMOND. Crystal Impact GbR, Bonn, Germany.

Bruker (2014). APEX2, SAINT and SADABS. Bruker AXS Inc., Madison, Wisconsin, USA.

Chifotides, H. T. \& Dunbar, K. R. (2013). Acc. Chem. Res. 46, 894906.

Furukawa, S., Reboul, J., Diring, S., Sumida, K. \& Kitagawa, S. (2014). Chem. Soc. Rev. 43, 5700-5734.

Groom, C. R., Bruno, I. J., Lightfoot, M. P. \& Ward, S. C. (2016). Acta Cryst. B72, 171-179.

Hanton, L. R., Hellyer, R. M. \& Spicer, M. D. (2006). Inorg. Chim. Acta, 359, 3659-3665.

Lee, E., Seo, J., Lee, S. S. \& Park, K.-M. (2012). Cryst. Growth Des. 12, 3834-3837.

Leong, W. L. \& Vittal, J. J. (2011). Chem. Rev. 111, 688-764. 
Liu, D., Chang, Y.-J. \& Lang, J.-P. (2011). CrystEngComm, 13, 18511857.

Matter, H., Nazaré, M., Güssregen, S., Will, D. W., Schreuder, H., Bauer, A., Urmann, M., Ritter, K., Wagner, M. \& Wehner, V. (2009). Angew. Chem. Int. Ed. 48, 2911-2916.

Moon, S.-H., Kang, Y. \& Park, K.-M. (2017a). Acta Cryst. E73, 15871589.

Moon, S.-H., Seo, J. \& Park, K.-M. (2017b). Acta Cryst. E73, 17001703.

Park, K.-M., Seo, J., Moon, S.-H. \& Lee, S. S. (2010). Cryst. Growth Des. 10, 4148-4154.
Seo, J., Moon, S.-T., Kim, J., Lee, S. S. \& Park, K.-M. (2003). Bull. Korean Chem. Soc. 24, 1393-1395.

Sheldrick, G. M. (2008). Acta Cryst. A64, 112-122.

Sheldrick, G. M. (2015). Acta Cryst. C71, 3-8.

Silva, P., Vilela, S. M. F., Tomé, J. P. C. \& Almeida Paz, F. A. (2015). Chem. Soc. Rev. 44, 6774-6803.

Wang, C., Zhang, T. \& Lin, W. (2012). Chem. Rev. 112, 1084-1104.

Westrip, S. P. (2010). J. Appl. Cryst. 43, 920-925.

Zheng, S.-R., Yang, Q.-Y., Yang, R., Pan, M., Cao, R. \& Su, C.-Y. (2009). Cryst. Growth Des. 9, 2341-2353. 


\section{supporting information}

Acta Cryst. (2017). E73, 1871-1874 [https://doi.org/10.1107/S205698901701619X]

\section{A one-dimensional Hg" coordination polymer based on bis(pyridin-3-ylmethyl)- sulfane}

\section{Suk-Hee Moon, Youngjin Kang and Ki-Min Park}

Computing details

Data collection: APEX2 (Bruker, 2014); cell refinement: SAINT (Bruker, 2014); data reduction: SAINT (Bruker, 2014); program(s) used to solve structure: SHELXS97 (Sheldrick, 2008); program(s) used to refine structure: SHELXL2014 (Sheldrick, 2015); molecular graphics: DIAMOND (Brandenburg, 2010); software used to prepare material for publication: SHELXTL (Sheldrick, 2008) and publCIF (Westrip, 2010).

catena-Poly[[dichloridomercury(II)]- $\mu$-bis(pyridin-3-ylmethyl)sulfane- $\left.\kappa^{2} N: N^{\prime}\right]$

\section{Crystal data}

$\left[\mathrm{HgCl}_{2}\left(\mathrm{C}_{12} \mathrm{H}_{12} \mathrm{~N}_{2} \mathrm{~S}\right)\right]$

$M_{r}=487.79$

Monoclinic, $P 2_{1} / n$

$a=10.4724(11) \AA$

$b=13.1128(14) \AA$

$c=10.8914$ (12) $\AA$

$\beta=100.1171(18)^{\circ}$

$V=1472.4(3) \AA^{3}$

$Z=4$

\section{Data collection}

Bruker SMART APEX CCD diffractometer

$\varphi$ and $\omega$ scans

Absorption correction: multi-scan

(SADABS; Bruker, 2014)

$T_{\min }=0.447, T_{\max }=0.746$

8706 measured reflections

\section{Refinement}

Refinement on $F^{2}$

Least-squares matrix: full

$R\left[F^{2}>2 \sigma\left(F^{2}\right)\right]=0.033$

$w R\left(F^{2}\right)=0.076$

$S=1.03$

3197 reflections

163 parameters

0 restraints
$F(000)=912$

$D_{\mathrm{x}}=2.200 \mathrm{Mg} \mathrm{m}^{-3}$

Mo $K \alpha$ radiation, $\lambda=0.71073 \AA$

Cell parameters from 9216 reflections

$\theta=2.5-28.2^{\circ}$

$\mu=10.94 \mathrm{~mm}^{-1}$

$T=298 \mathrm{~K}$

Plate, colorless

$0.45 \times 0.40 \times 0.30 \mathrm{~mm}$

3197 independent reflections

2413 reflections with $I>2 \sigma(I)$

$R_{\text {int }}=0.047$

$\theta_{\max }=27.0^{\circ}, \theta_{\min }=2.5^{\circ}$

$h=-13 \rightarrow 11$

$k=-16 \rightarrow 10$

$l=-13 \rightarrow 13$

Hydrogen site location: inferred from neighbouring sites

$\mathrm{H}$-atom parameters constrained

$w=1 /\left[\sigma^{2}\left(F_{\mathrm{o}}^{2}\right)+(0.0315 P)^{2}\right]$

where $P=\left(F_{\mathrm{o}}^{2}+2 F_{\mathrm{c}}{ }^{2}\right) / 3$

$(\Delta / \sigma)_{\max }<0.001$

$\Delta \rho_{\max }=0.62 \mathrm{e} \AA^{-3}$

$\Delta \rho_{\min }=-1.62$ e $\AA^{-3}$ 


\section{Special details}

Geometry. All esds (except the esd in the dihedral angle between two 1.s. planes) are estimated using the full covariance matrix. The cell esds are taken into account individually in the estimation of esds in distances, angles and torsion angles; correlations between esds in cell parameters are only used when they are defined by crystal symmetry. An approximate (isotropic) treatment of cell esds is used for estimating esds involving l.s. planes.

Fractional atomic coordinates and isotropic or equivalent isotropic displacement parameters $\left(\AA^{2}\right)$

\begin{tabular}{lllll}
\hline & $x$ & $y$ & $z$ & $U_{\text {iso }} / U_{\text {eq }}$ \\
\hline Hg1 & $0.62237(2)$ & $0.33807(2)$ & $0.42736(2)$ & $0.04266(10)$ \\
C11 & $0.54910(16)$ & $0.36205(14)$ & $0.21164(15)$ & $0.0559(4)$ \\
C12 & $0.75516(17)$ & $0.37587(15)$ & $0.62207(16)$ & $0.0633(5)$ \\
S1 & $-0.06074(16)$ & $0.35443(14)$ & $0.33023(18)$ & $0.0598(5)$ \\
N1 & $0.4127(5)$ & $0.3509(4)$ & $0.4938(4)$ & $0.0416(12)$ \\
N2 & $-0.1449(4)$ & $0.6533(4)$ & $0.0651(4)$ & $0.0408(12)$ \\
C1 & $0.4063(6)$ & $0.3940(5)$ & $0.6036(6)$ & $0.0467(15)$ \\
H1 & 0.4823 & 0.4178 & 0.6525 & $0.056^{*}$ \\
C2 & $0.2918(7)$ & $0.4044(5)$ & $0.6469(6)$ & $0.0551(17)$ \\
H2 & 0.2897 & 0.4384 & 0.7215 & $0.066^{*}$ \\
C3 & $0.1800(7)$ & $0.3642(5)$ & $0.5789(7)$ & $0.0537(17)$ \\
H3 & 0.1018 & 0.3697 & 0.6079 & $0.064^{*}$ \\
C4 & $0.1849(6)$ & $0.3153(4)$ & $0.4666(6)$ & $0.0414(14)$ \\
C5 & $0.3037(6)$ & $0.3121(4)$ & $0.4282(6)$ & $0.0425(14)$ \\
H5 & 0.3081 & 0.2812 & 0.3523 & $0.051^{*}$ \\
C6 & $0.0678(6)$ & $0.2659(5)$ & $0.3898(7)$ & $0.0545(17)$ \\
H6A & 0.0341 & 0.2151 & 0.4405 & $0.065^{*}$ \\
H6B & 0.0944 & 0.2307 & 0.3201 & $0.065^{*}$ \\
C7 & $0.0153(6)$ & $0.4169(5)$ & $0.2132(6)$ & $0.0462(15)$ \\
H7A & 0.0896 & 0.4559 & 0.2536 & $0.055^{*}$ \\
H7B & 0.0457 & 0.3660 & 0.1605 & $0.055^{*}$ \\
C8 & $-0.0790(5)$ & $0.4860(5)$ & $0.1354(5)$ & $0.0403(13)$ \\
C9 & $-0.0627(5)$ & $0.5903(4)$ & $0.1356(5)$ & $0.0379(13)$ \\
H9 & 0.0090 & 0.6181 & 0.1873 & $0.045^{*}$ \\
C10 & $-0.2482(6)$ & $0.6139(5)$ & $-0.0097(6)$ & $0.0464(15)$ \\
H10 & -0.3048 & 0.6575 & -0.0601 & $0.056^{*}$ \\
C11 & $-0.2726(6)$ & $0.5132(5)$ & $-0.0141(6)$ & $0.0574(17)$ \\
H11 & -0.3457 & 0.4882 & -0.0663 & $0.069^{*}$ \\
C12 & $-0.1897(6)$ & $0.4473(5)$ & $0.0583(6)$ & $0.0503(16)$ \\
H12 & -0.2068 & 0.3776 & 0.0563 & $0.060^{*}$ \\
& & & &
\end{tabular}

Atomic displacement parameters $\left(\AA^{2}\right)$

\begin{tabular}{lllllll}
\hline & $U^{11}$ & $U^{22}$ & $U^{33}$ & $U^{12}$ & $U^{13}$ & $U^{23}$ \\
\hline Hg1 & $0.03985(13)$ & $0.04678(16)$ & $0.03812(15)$ & $0.00356(11)$ & $-0.00208(9)$ & $-0.00140(12)$ \\
C11 & $0.0515(9)$ & $0.0733(12)$ & $0.0385(9)$ & $0.0022(8)$ & $-0.0039(7)$ & $-0.0006(8)$ \\
C12 & $0.0602(10)$ & $0.0726(12)$ & $0.0482(10)$ & $-0.0080(9)$ & $-0.0154(8)$ & $-0.0059(9)$ \\
S1 & $0.0373(8)$ & $0.0776(13)$ & $0.0666(12)$ & $0.0107(8)$ & $0.0149(8)$ & $0.0249(10)$ \\
N1 & $0.041(3)$ & $0.048(3)$ & $0.035(3)$ & $0.011(2)$ & $0.006(2)$ & $-0.002(2)$
\end{tabular}




$\begin{array}{lllllll}\mathrm{N} 2 & 0.039(3) & 0.042(3) & 0.039(3) & -0.004(2) & 0.000(2) & 0.002(2) \\ \text { C1 } & 0.047(3) & 0.046(4) & 0.046(4) & -0.003(3) & 0.004(3) & 0.001(3) \\ \text { C2 } & 0.063(4) & 0.061(5) & 0.045(4) & 0.004(3) & 0.021(3) & -0.009(3) \\ \text { C3 } & 0.056(4) & 0.053(4) & 0.057(4) & -0.001(3) & 0.024(3) & -0.002(3) \\ \text { C4 } & 0.043(3) & 0.033(3) & 0.049(4) & 0.011(2) & 0.009(3) & 0.012(3) \\ \text { C5 } & 0.044(3) & 0.047(4) & 0.037(3) & 0.010(3) & 0.007(3) & -0.003(3) \\ \text { C6 } & 0.044(4) & 0.047(4) & 0.071(5) & -0.001(3) & 0.008(3) & 0.017(3) \\ \text { C7 } & 0.039(3) & 0.051(4) & 0.051(4) & 0.000(3) & 0.013(3) & 0.006(3) \\ \text { C8 } & 0.043(3) & 0.047(4) & 0.031(3) & 0.001(3) & 0.006(2) & -0.003(3) \\ \text { C9 } & 0.032(3) & 0.045(4) & 0.036(3) & -0.005(2) & 0.003(2) & -0.002(3) \\ \text { C10 } & 0.040(3) & 0.055(4) & 0.039(4) & -0.005(3) & -0.006(3) & 0.001(3) \\ \text { C11 } & 0.056(4) & 0.055(4) & 0.053(4) & -0.013(3) & -0.011(3) & -0.004(3) \\ \text { C12 } & 0.051(4) & 0.040(4) & 0.056(4) & -0.014(3) & -0.002(3) & -0.003(3)\end{array}$

Geometric parameters $\left(\AA,{ }^{\circ}\right)$

\begin{tabular}{|c|c|c|c|}
\hline $\mathrm{Hg} 1-\mathrm{Cl1}$ & $2.3610(16)$ & $\mathrm{C} 4-\mathrm{C} 5$ & $1.381(8)$ \\
\hline $\mathrm{Hg} 1-\mathrm{Cl} 2$ & $2.3751(16)$ & $\mathrm{C} 4-\mathrm{C} 6$ & $1.504(9)$ \\
\hline $\mathrm{Hg} 1-\mathrm{N} 2^{\mathrm{i}}$ & $2.434(5)$ & $\mathrm{C} 5-\mathrm{H} 5$ & 0.9300 \\
\hline $\mathrm{Hg} 1-\mathrm{N} 1$ & $2.436(5)$ & C6-H6A & 0.9700 \\
\hline $\mathrm{S} 1-\mathrm{C} 6$ & $1.810(6)$ & C6-H6B & 0.9700 \\
\hline $\mathrm{S} 1-\mathrm{C} 7$ & $1.813(6)$ & $\mathrm{C} 7-\mathrm{C} 8$ & $1.490(8)$ \\
\hline $\mathrm{N} 1-\mathrm{C} 5$ & $1.335(8)$ & $\mathrm{C} 7-\mathrm{H} 7 \mathrm{~A}$ & 0.9700 \\
\hline $\mathrm{N} 1-\mathrm{C} 1$ & $1.336(7)$ & $\mathrm{C} 7-\mathrm{H} 7 \mathrm{~B}$ & 0.9700 \\
\hline $\mathrm{N} 2-\mathrm{C} 9$ & $1.334(7)$ & $\mathrm{C} 8-\mathrm{C} 9$ & $1.378(8)$ \\
\hline $\mathrm{N} 2-\mathrm{C} 10$ & $1.339(7)$ & $\mathrm{C} 8-\mathrm{C} 12$ & $1.402(8)$ \\
\hline $\mathrm{N} 2-\mathrm{Hg} 1^{\mathrm{ii}}$ & $2.434(5)$ & C9-H9 & 0.9300 \\
\hline $\mathrm{C} 1-\mathrm{C} 2$ & $1.370(8)$ & $\mathrm{C} 10-\mathrm{C} 11$ & $1.345(9)$ \\
\hline $\mathrm{C} 1-\mathrm{H} 1$ & 0.9300 & $\mathrm{C} 10-\mathrm{H} 10$ & 0.9300 \\
\hline $\mathrm{C} 2-\mathrm{C} 3$ & $1.376(10)$ & $\mathrm{C} 11-\mathrm{C} 12$ & $1.372(9)$ \\
\hline $\mathrm{C} 2-\mathrm{H} 2$ & 0.9300 & C11-H11 & 0.9300 \\
\hline $\mathrm{C} 3-\mathrm{C} 4$ & $1.390(9)$ & $\mathrm{C} 12-\mathrm{H} 12$ & 0.9300 \\
\hline $\mathrm{C} 3-\mathrm{H} 3$ & 0.9300 & & \\
\hline $\mathrm{Cl1}-\mathrm{Hg} 1-\mathrm{Cl} 2$ & $153.86(7)$ & $\mathrm{C} 4-\mathrm{C} 6-\mathrm{S} 1$ & $113.9(4)$ \\
\hline $\mathrm{Cl1}-\mathrm{Hg} 1-\mathrm{N} 2^{\mathrm{i}}$ & $100.29(12)$ & $\mathrm{C} 4-\mathrm{C} 6-\mathrm{H} 6 \mathrm{~A}$ & 108.8 \\
\hline $\mathrm{Cl} 2-\mathrm{Hg} 1-\mathrm{N} 2^{\mathrm{i}}$ & $98.03(12)$ & $\mathrm{S} 1-\mathrm{C} 6-\mathrm{H} 6 \mathrm{~A}$ & 108.8 \\
\hline $\mathrm{Cl1}-\mathrm{Hg} 1-\mathrm{N} 1$ & $97.69(12)$ & $\mathrm{C} 4-\mathrm{C} 6-\mathrm{H} 6 \mathrm{~B}$ & 108.8 \\
\hline $\mathrm{Cl} 2-\mathrm{Hg} 1-\mathrm{N} 1$ & $97.91(13)$ & $\mathrm{S} 1-\mathrm{C} 6-\mathrm{H} 6 \mathrm{~B}$ & 108.8 \\
\hline $\mathrm{N} 22^{\mathrm{i}}-\mathrm{Hg} 1-\mathrm{N} 1$ & $98.39(16)$ & $\mathrm{H} 6 \mathrm{~A}-\mathrm{C} 6-\mathrm{H} 6 \mathrm{~B}$ & 107.7 \\
\hline $\mathrm{C} 6-\mathrm{S} 1-\mathrm{C} 7$ & $98.7(3)$ & $\mathrm{C} 8-\mathrm{C} 7-\mathrm{S} 1$ & $110.2(4)$ \\
\hline $\mathrm{C} 5-\mathrm{N} 1-\mathrm{C} 1$ & $117.9(5)$ & $\mathrm{C} 8-\mathrm{C} 7-\mathrm{H} 7 \mathrm{~A}$ & 109.6 \\
\hline $\mathrm{C} 5-\mathrm{N} 1-\mathrm{Hg} 1$ & $123.0(4)$ & $\mathrm{S} 1-\mathrm{C} 7-\mathrm{H} 7 \mathrm{~A}$ & 109.6 \\
\hline $\mathrm{C} 1-\mathrm{N} 1-\mathrm{Hg} 1$ & $119.0(4)$ & $\mathrm{C} 8-\mathrm{C} 7-\mathrm{H} 7 \mathrm{~B}$ & 109.6 \\
\hline $\mathrm{C} 9-\mathrm{N} 2-\mathrm{C} 10$ & $118.8(5)$ & $\mathrm{S} 1-\mathrm{C} 7-\mathrm{H} 7 \mathrm{~B}$ & 109.6 \\
\hline $\mathrm{C} 9-\mathrm{N} 2-\mathrm{Hg} 1^{\mathrm{ii}}$ & $123.3(4)$ & $\mathrm{H} 7 \mathrm{~A}-\mathrm{C} 7-\mathrm{H} 7 \mathrm{~B}$ & 108.1 \\
\hline $\mathrm{C} 10-\mathrm{N} 2-\mathrm{Hg} 1^{\mathrm{ii}}$ & $117.9(4)$ & $\mathrm{C} 9-\mathrm{C} 8-\mathrm{C} 12$ & $116.7(6)$ \\
\hline $\mathrm{N} 1-\mathrm{C} 1-\mathrm{C} 2$ & $122.3(6)$ & $\mathrm{C} 9-\mathrm{C} 8-\mathrm{C} 7$ & $122.2(5)$ \\
\hline
\end{tabular}




\begin{tabular}{|c|c|c|c|}
\hline $\mathrm{N} 1-\mathrm{C} 1-\mathrm{H} 1$ & 118.8 & $\mathrm{C} 12-\mathrm{C} 8-\mathrm{C} 7$ & $121.0(6)$ \\
\hline $\mathrm{C} 2-\mathrm{C} 1-\mathrm{H} 1$ & 118.8 & $\mathrm{~N} 2-\mathrm{C} 9-\mathrm{C} 8$ & $123.1(5)$ \\
\hline $\mathrm{C} 1-\mathrm{C} 2-\mathrm{C} 3$ & $119.3(6)$ & $\mathrm{N} 2-\mathrm{C} 9-\mathrm{H} 9$ & 118.4 \\
\hline $\mathrm{C} 1-\mathrm{C} 2-\mathrm{H} 2$ & 120.3 & $\mathrm{C} 8-\mathrm{C} 9-\mathrm{H} 9$ & 118.4 \\
\hline $\mathrm{C} 3-\mathrm{C} 2-\mathrm{H} 2$ & 120.3 & $\mathrm{~N} 2-\mathrm{C} 10-\mathrm{C} 11$ & $121.9(6)$ \\
\hline $\mathrm{C} 2-\mathrm{C} 3-\mathrm{C} 4$ & $119.4(6)$ & $\mathrm{N} 2-\mathrm{C} 10-\mathrm{H} 10$ & 119.0 \\
\hline $\mathrm{C} 2-\mathrm{C} 3-\mathrm{H} 3$ & 120.3 & $\mathrm{C} 11-\mathrm{C} 10-\mathrm{H} 10$ & 119.0 \\
\hline $\mathrm{C} 4-\mathrm{C} 3-\mathrm{H} 3$ & 120.3 & $\mathrm{C} 10-\mathrm{C} 11-\mathrm{C} 12$ & $120.1(6)$ \\
\hline $\mathrm{C} 5-\mathrm{C} 4-\mathrm{C} 3$ & $117.0(6)$ & $\mathrm{C} 10-\mathrm{C} 11-\mathrm{H} 11$ & 120.0 \\
\hline $\mathrm{C} 5-\mathrm{C} 4-\mathrm{C} 6$ & $120.6(6)$ & $\mathrm{C} 12-\mathrm{C} 11-\mathrm{H} 11$ & 120.0 \\
\hline $\mathrm{C} 3-\mathrm{C} 4-\mathrm{C} 6$ & $122.4(6)$ & $\mathrm{C} 11-\mathrm{C} 12-\mathrm{C} 8$ & $119.3(6)$ \\
\hline $\mathrm{N} 1-\mathrm{C} 5-\mathrm{C} 4$ & $123.9(6)$ & $\mathrm{C} 11-\mathrm{C} 12-\mathrm{H} 12$ & 120.4 \\
\hline $\mathrm{N} 1-\mathrm{C} 5-\mathrm{H} 5$ & 118.1 & $\mathrm{C} 8-\mathrm{C} 12-\mathrm{H} 12$ & 120.4 \\
\hline $\mathrm{C} 4-\mathrm{C} 5-\mathrm{H} 5$ & 118.1 & & \\
\hline $\mathrm{C} 5-\mathrm{N} 1-\mathrm{C} 1-\mathrm{C} 2$ & $-3.7(9)$ & $\mathrm{C} 6-\mathrm{S} 1-\mathrm{C} 7-\mathrm{C} 8$ & $172.1(5)$ \\
\hline $\mathrm{Hg} 1-\mathrm{N} 1-\mathrm{C} 1-\mathrm{C} 2$ & $179.6(5)$ & $\mathrm{S} 1-\mathrm{C} 7-\mathrm{C} 8-\mathrm{C} 9$ & $114.8(5)$ \\
\hline $\mathrm{N} 1-\mathrm{C} 1-\mathrm{C} 2-\mathrm{C} 3$ & $3.8(10)$ & $\mathrm{S} 1-\mathrm{C} 7-\mathrm{C} 8-\mathrm{C} 12$ & $-65.0(7)$ \\
\hline $\mathrm{C} 1-\mathrm{C} 2-\mathrm{C} 3-\mathrm{C} 4$ & $-1.1(10)$ & $\mathrm{C} 10-\mathrm{N} 2-\mathrm{C} 9-\mathrm{C} 8$ & $-0.1(8)$ \\
\hline $\mathrm{C} 2-\mathrm{C} 3-\mathrm{C} 4-\mathrm{C} 5$ & $-1.4(9)$ & $\mathrm{Hg} 1{ }^{\mathrm{ii}}-\mathrm{N} 2-\mathrm{C} 9-\mathrm{C} 8$ & $176.9(4)$ \\
\hline $\mathrm{C} 2-\mathrm{C} 3-\mathrm{C} 4-\mathrm{C} 6$ & $177.5(6)$ & $\mathrm{C} 12-\mathrm{C} 8-\mathrm{C} 9-\mathrm{N} 2$ & $-1.4(9)$ \\
\hline $\mathrm{C} 1-\mathrm{N} 1-\mathrm{C} 5-\mathrm{C} 4$ & $0.9(9)$ & $\mathrm{C} 7-\mathrm{C} 8-\mathrm{C} 9-\mathrm{N} 2$ & $178.9(5)$ \\
\hline $\mathrm{Hg} 1-\mathrm{N} 1-\mathrm{C} 5-\mathrm{C} 4$ & $177.5(4)$ & $\mathrm{C} 9-\mathrm{N} 2-\mathrm{C} 10-\mathrm{C} 11$ & $1.2(9)$ \\
\hline $\mathrm{C} 3-\mathrm{C} 4-\mathrm{C} 5-\mathrm{N} 1$ & $1.6(9)$ & $\mathrm{Hg} 1^{\mathrm{ii}}-\mathrm{N} 2-\mathrm{C} 10-\mathrm{C} 11$ & $-175.9(5)$ \\
\hline $\mathrm{C} 6-\mathrm{C} 4-\mathrm{C} 5-\mathrm{N} 1$ & $-177.4(5)$ & $\mathrm{N} 2-\mathrm{C} 10-\mathrm{C} 11-\mathrm{C} 12$ & $-0.7(10)$ \\
\hline $\mathrm{C} 5-\mathrm{C} 4-\mathrm{C} 6-\mathrm{S} 1$ & $-117.0(5)$ & $\mathrm{C} 10-\mathrm{C} 11-\mathrm{C} 12-\mathrm{C} 8$ & $-0.8(10)$ \\
\hline $\mathrm{C} 3-\mathrm{C} 4-\mathrm{C} 6-\mathrm{S} 1$ & $64.1(7)$ & $\mathrm{C} 9-\mathrm{C} 8-\mathrm{C} 12-\mathrm{C} 11$ & $1.8(9)$ \\
\hline $\mathrm{C} 7-\mathrm{S} 1-\mathrm{C} 6-\mathrm{C} 4$ & $71.9(5)$ & $\mathrm{C} 7-\mathrm{C} 8-\mathrm{C} 12-\mathrm{C} 11$ & $-178.5(6)$ \\
\hline
\end{tabular}

Symmetry codes: (i) $-x+1 / 2, y-1 / 2,-z+1 / 2$; (ii) $-x+1 / 2, y+1 / 2,-z+1 / 2$.

Hydrogen-bond geometry $\left(\AA,{ }^{\circ}\right)$

$\mathrm{Cg} 2$ is the centroid of the N2/C8-C12 ring.

\begin{tabular}{lllll}
\hline$D-\mathrm{H} \cdots A$ & $D-\mathrm{H}$ & $\mathrm{H} \cdots A$ & $D \cdots A$ & $D-\mathrm{H} \cdots A$ \\
\hline $\mathrm{C} 10-\mathrm{H} 10 \cdots \mathrm{C} 11^{\mathrm{iii}}$ & 0.93 & 2.80 & $3.526(6)$ & 136 \\
$\mathrm{C} 2-\mathrm{H} 2 \cdots C g 2^{\mathrm{iv}}$ & 0.93 & 2.89 & $3.689(7)$ & 145 \\
\hline
\end{tabular}

Symmetry codes: (iii) $-x,-y+1,-z$; (iv) $-x,-y+1,-z+1$. 